\title{
Circumferential Margin Status by MRI
}

National Cancer Institute

\section{Source}

National Cancer Institute. Circumferential Margin Status by MRI. NCI Thesaurus. Code C157076.

The determination by MRI of the presence or absence of neoplastic tissue outside of a circumferential resection margin. 